\title{
FACE SKETCH GENERATION USING EVOLUTIONARY COMPUTING
}

\author{
N K Bansode ${ }^{1}$ and P K Sinha ${ }^{2}$ \\ ${ }^{1}$ Department of Computer Engineering, College of Engineering, Pune
}

\begin{abstract}
In this paper, an evolutionary genetic algorithm is used to generate face sketch from the face description. Face sketch generation without face image is extremely important for the law enforcement agencies. The genetic algorithm is used for generating face sketch through several iterations of the algorithm. The face image description is captured through graphical user interface just by clicking options for each face features. Face features are used to extract face images and generate initial population for the genetic algorithm. Genetic operators such as selection, crossover and mutation are used for next generation of the population. The Genetic algorithm cycle is repeated until the user is satisfied with face sketch generated. The novelty of the paper includes face sketch generation from face image description. The result shows that evolutionary based technique for sketch generation produces the desired face sketch.
\end{abstract}

\section{KEYWORDS}

Evolutionary Computing, Genetic Algorithm, Face Sketch Generation

\section{INTRODUCTION}

Face plays an important role in person identification and conveys a verity of demographic information like age, gender, and emotions. We can recognize a familiar person and remember for several years. There are several applications of automatic face recognition such face authentication, face movement tracking, security, and surveillance.

In Investigation, witness or victim of the crime provides the description of an attacker or any other source of information related to crime. Witness plays a very important role to give valuable information regarding the crime. Sometimes the attacker face image is not available in such cases, an artist help is taken to generate face sketch from the description given by the witness. Employing artist for face sketch generation is time consuming and tedious tasks. The cognitive interview process is used to obtain information from a witness of the crime regarding the facial description of the suspect.

To enable a computer to generate face sketch from the description involves an automation of conceptual sketching. Face composite systems were developed as alternate systems to the sketch artist. The face composite generation consists of a selection of the face features matching with target face and assembling together in the face frame. In the past several years, face composite systems were developed with the use of new technology for composite generation. The problem with face composite system is that, the limited number of the face features supported for face composite generation. The advanced face composite systems consist of large dataset of the facial features.

The recent face composites are generated using evolutionary genetic algorithm [1]. The evolutionary algorithm generates a variety of the faces by evolving face through several 
generations. The user (witness) selects the best matching face in the current population and new faces are generated through the process of face evolutions. The process stops when the generated faces are similar to the target face.

\section{RELATED WORK}

Face sketch generation systems developed in the past from sketch artist to the modern intelligent algorithm such as a genetic algorithm. The face composite systems developed using technological support available at the time of development of the composite systems. Due to technological advancement, a variety of the face composite systems developed over the period of the time [2]. The study of such systems presented in the following paragraphs.

Xiaoou Tang and Xiaogang Wang [19] presented photo retrieval system using sketch drawing. Face photo recognized using sketches. Face features such as shape and texture calculated by eigen transformations. Hao Wang and Kangqiao Wang [6] used feature extraction and image based face drawing. Hough transforms used for face component detection such as eyes and intensity valley information to locate the pair of iris. Xiaoou Tang and Xiaogang Wang [20] described face recognition system using face sketches. A database of face photo and sketches of 188 people is used for photo retrieval. The image of face photo and sketch represents the different form of the image. Photo image represented by grayscale values and texture information, while sketches presented only by the grayscale values. In order to match sketch with the photo, the photo image converted into the sketch image. i.e. a database of photo image transformed into a database of sketch images. The eigenface recognition used to recognize face sketch in the database.

Hong Chen et al. [5,8,9] attempted to generate example based composite sketching of human portraits. The method for drawing face composite similar to the method used by the artist used for drawing of the picture. Fan Yang [3] presented non-parametric generation of example based human facial sketch. The conditional distribution of pixels in sketch image used to generate sketch. Hao Wang [7] attempted to draw a face using active shape models and parametric morphing.

Futoshi Sugimoto et al [4] presented drawing of a facial image in users mind using psychometric space model of the face. An image in user mind represented as psychological space model and image sketch considered into the different model of drawing. The genetic algorithm used to search image. Fuzzy reasoning is used to calculate the fitness of images generated by the genetic algorithm [15]. The process is repeated until the user gets satisfied or maximum number of generations are completed. Junji Nishino and Tomonori Kameyama [10] explained the process of caricature drawing using linguistic variables for the face features. Mayada $\mathrm{F}$ and Abdul Halim et al [11] described a system for facial composite generation using the genetic algorithm. The system consists of two step process. In step one, a database of facial part is created. In the second step, the genetic algorithm is used for reconstructing the facial composite image likeness to the facial composite image in mind of the witness. The recognition based strategy used to recognize the image rather than to recall the image. Additional tools for painting, smoothing, and sharpening used to enhance quality of the facial image.

Masashi Yamada et al [12] used the genetic algorithm to draw a logo. The picture of logo consists of one string and two images. Stuart Gibson et al [16] presented a facial composite system using an evolutionary algorithm. Global and local model for face features used for drawing face composite. Shape and texture for training images are derived and treated as the appearance model for the face. A witness is presented with virtual faces and allowed to determine likeness and ranking of each face by comparing with the target face. Three variants of evolutionary algorithm 
used and their performance measured using the virtual witness. Pong C, Yuen and C H Man [13] performed an experiment for human face searching using the face sketch images.

The literature survey for face composite generation indicates that several face composite systems were developed and the performance of these face composite systems was low. In this paper, we have implemented new approach for face sketch generation algorithm to generate face sketch from face image description.

\section{Methodology}

The face sketch is generated using the process of the evolution. The genetic algorithm evolves the faces through several generations. The genetic algorithm is a search and optimization technique based on Darwin's principal of the "survival of the fittest". Face sketch generation system based on the genetic algorithm is an advanced process of the sketch generation which evolves the face using genetic operators. The Genetic algorithm is described in the following sections.

\subsection{Genetic Algorithm}

Genetic Algorithms are an evolutionary procedure that finds the solution to problems using the mechanics of natural selection. Genetic algorithms are used in the problem where the finding solution is difficult, but due to the probabilistic nature these algorithms gives optimal solutions. In the cycle of the genetic algorithm as shown in figure 1, it starts with an initial set of random solutions called population. Each individual in the population is called as chromosome, representing the solution to the problem to solve. During each generation, the chromosomes are evaluated using some measure of fitness [21]. This fitness of individual solution string is used to create the next generation, a new chromosome are formed by three essential operations: selection, crossover, mutation. The process of the genetic algorithm cycle is shown in Figure 1.

\subsection{Genetic Operators}

Selection is a process in which individual strings are copied according to their objective (fitness) function values. Copying strings according to their fitness value uses means that strings with a higher fitness value will have a higher probability contributing one or more offspring in the next generation. The crossover is a process of merging two chromosomes from current generation to from two similar offspring's. The mutation is a process of modifying a chromosome and occasionally one or more bits of a string are altered while the process is being performed. The flowchart of the genetic algorithm is shown in Figure 2

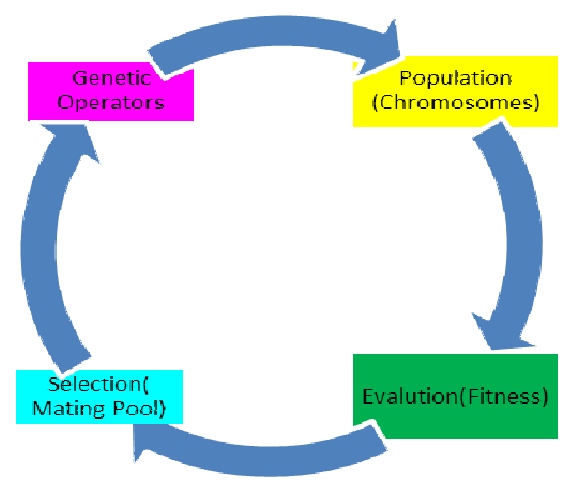

Figure 1: Genetic Algorithm Cycle 
International Journal on Soft Computing (IJSC) Vol.7, No. 4, November 2016

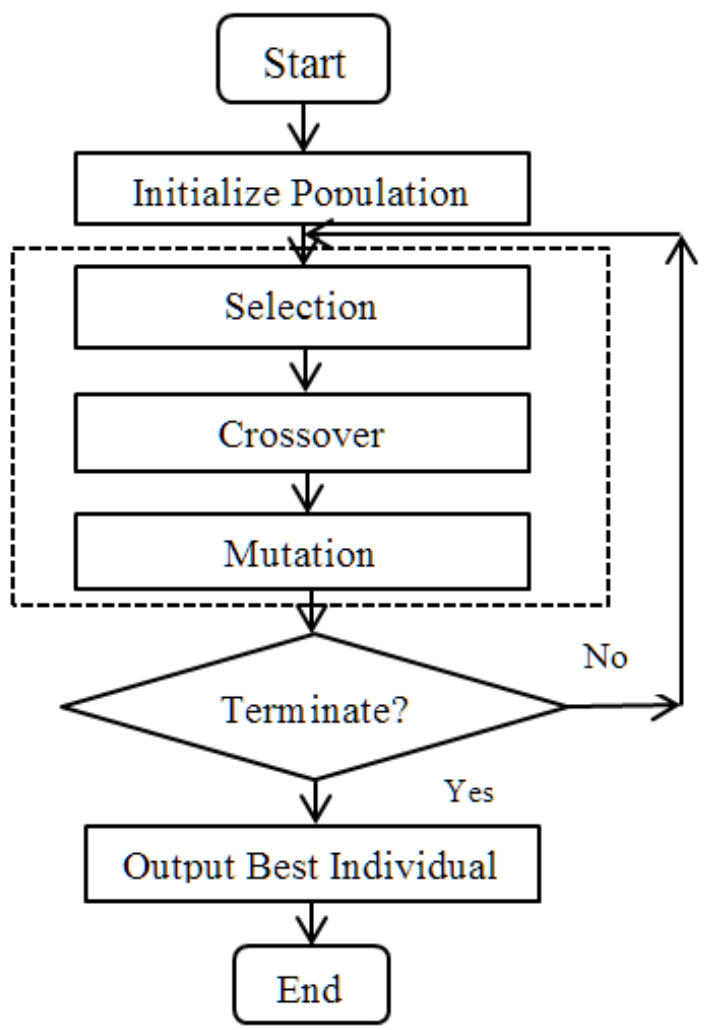

Figure 2: Genetic Algorithm flowchart

Table 1: Face features description parameters

\begin{tabular}{|l|l|l|l|l|}
\hline Sr. No. & Features & Male & Female & \\
\hline 1 & Gender & Child & Young & Old \\
\hline 2 & Age Group & Ellipse & Circle & Oval, Square, Triangle \\
\hline 3 & Face Shape & Small & Normal & Large \\
\hline 4 & Left Eye brow & Small & Normal & Large \\
\hline 5 & Right Eyebrow & Thin & Medium & Large \\
\hline 6 & Left Eye & Small & Normal & Large \\
\hline 7 & Right Eye & Small & Normal & Large \\
\hline 8 & Nose & Thin & Medium & Large \\
\hline 9 & Mouth & & & \\
\hline
\end{tabular}




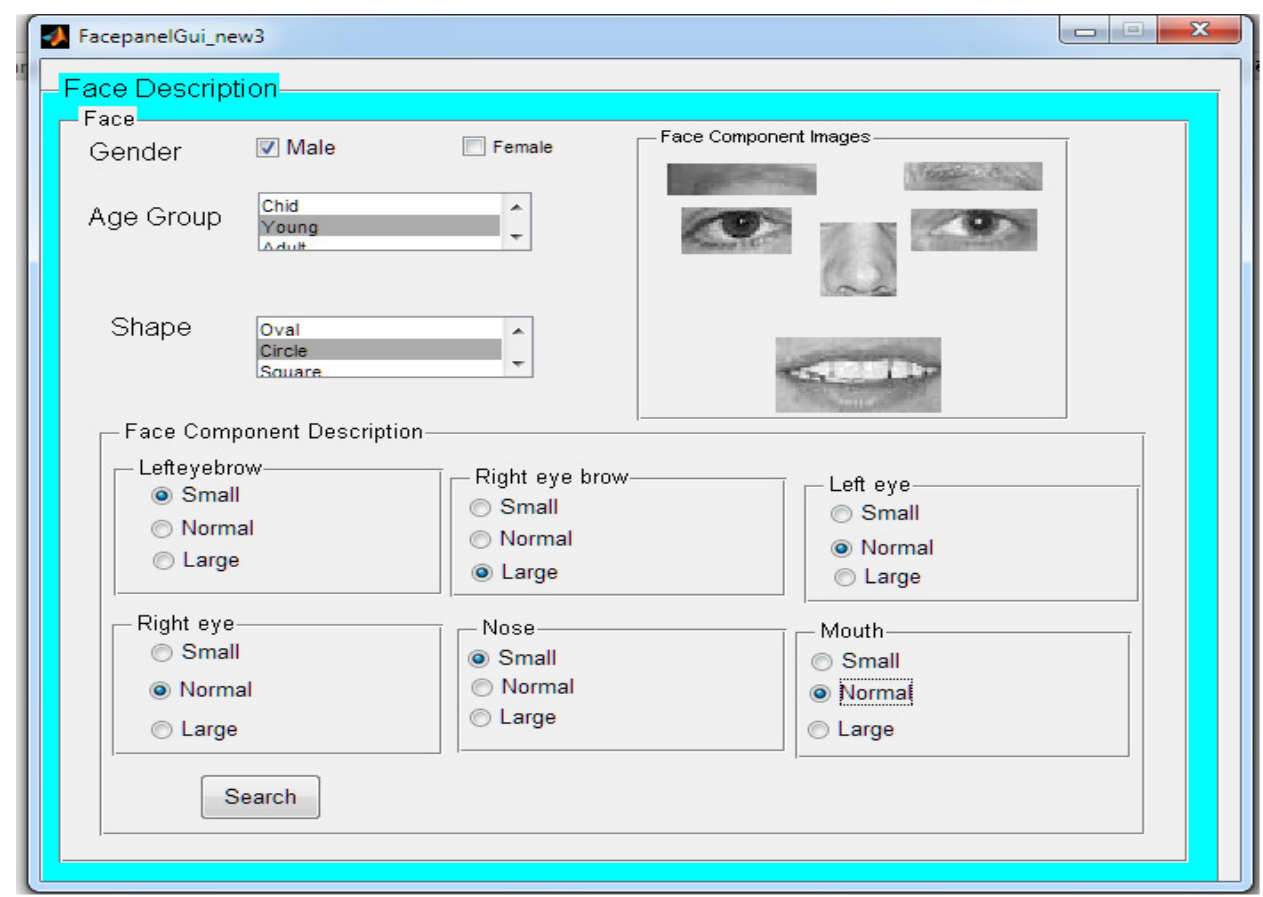

Figure 3 : Graphical user interface for face description

\subsection{Face Sketch Generation Algorithm}

The face sketch is generated using evolutionary genetic algorithm based on the facial feature description provided by the user. Table 1 shows the face features description and the possible parameter values. The graphical user interface is designed as shown in Figure 3 for capturing the facial parameters. The facial description entered through the GUI is used to extract faces which are resemble to the feature descriptions. The faces collected from the description used as initial population for the genetic algorithm. The genetic algorithm works on these faces is given below:

\section{Genetic algorithm for Sketch Generation}

// This algorithm generates the face composite from the face image

// Input : Face Image

// Output : Face Sketch

// Input parameters: Population Size, Crossover Rate, Mutation Rate, Max. Number of Generations

\section{Procedure}

1. Begin

2. Start Generation $(t<0)$

3. Initialize Face Population (t)

4. While (Not Termination Condition)

$5 . \quad$ begin

6. $\quad \mathrm{t}<-\mathrm{t}+1 \quad$ // Generation= Generation +1

7. Select $\mathrm{p}(\mathrm{t})$ from $\mathrm{p}(\mathrm{t}-1) \quad / /$ Select the parent faces from the population

8. Crossover $\mathrm{p}(\mathrm{t}) \quad / /$ Crossover the face to produce new faces 
International Journal on Soft Computing (IJSC) Vol.7, No. 4, November 2016

9.

10.

11. end

12. End

13. End
// Modify the face (genotype)

// Find the fitness with face with the target face // End of Generation // End of Maximum Number of Generations // End Begin

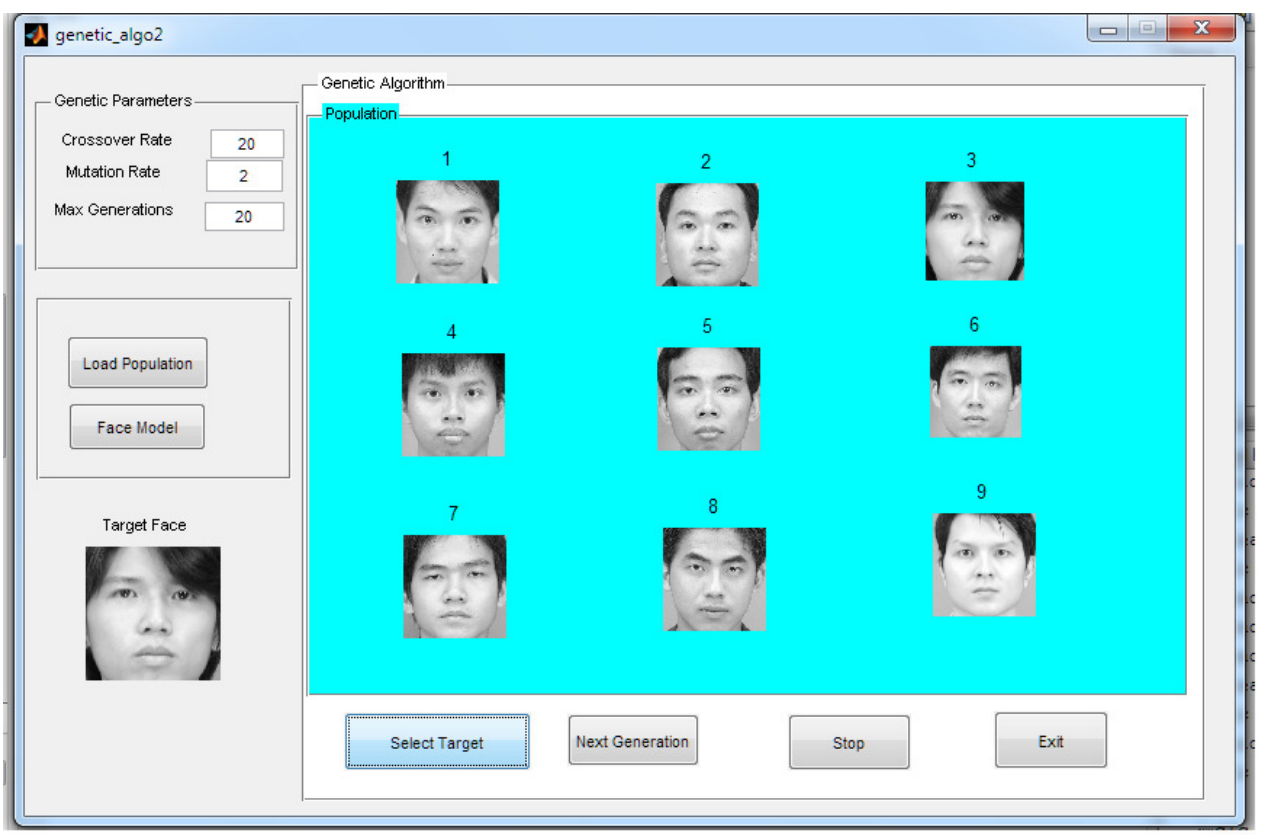

Figure 4: Face Skecth Generation (Initial Young Population)

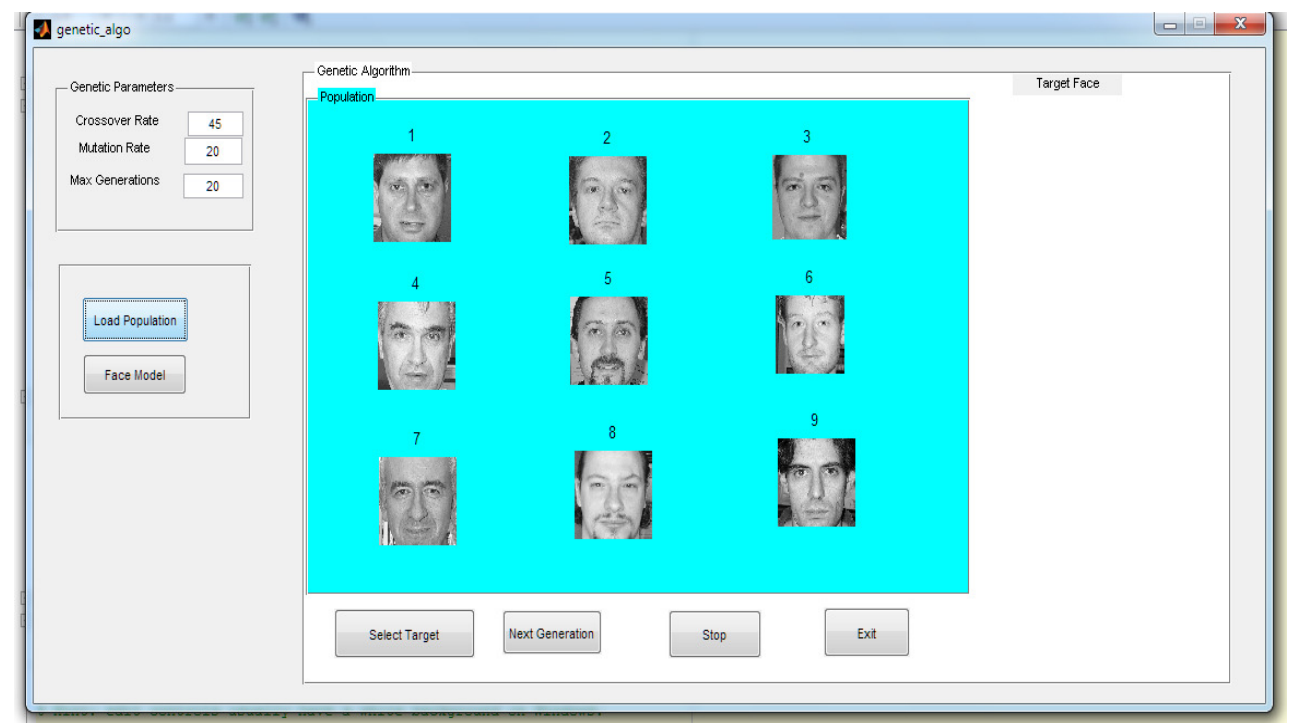

Figure 5: Face sketch Generation (Initial Old Populations) 


\section{Results}

The face sketch generation from the face features description is performed based on the evolutionary genetic algorithm. This system developed for automatic face sketch generation similar to the sketch artists. Face sketch generation for two types of population such as young and old population is implemented as shown in Figure 4 for young population and Figure 5 for old population. The genetic algorithm parameters such as population size, crossover rate and mutation rate are specified for each type of the population. The population after the 20 generations are converged and shown in Figure 6 for young population. The mean square error is measured for every generation and shown in Table 2 and 3. The two face datasets used for sketch generations [22, 23]. Figure 7 and 8 shows graph for the mean square error in each generation. The average mean square error is reduced in each generation.

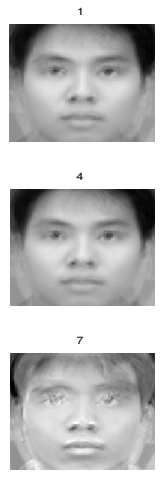

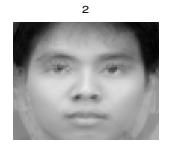
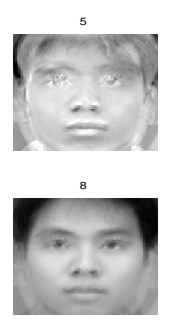
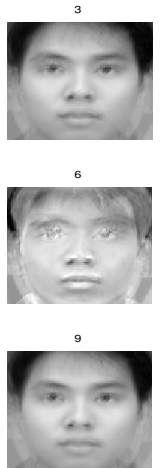

Figure 6: Population after 20 generations

Table 2: Final Population after 20 Generation (Young Population)

\begin{tabular}{|l|l|l|l|l|l|l|l|l|l|}
\hline $\begin{array}{l}\text { Facel } \\
\text { Gen. }\end{array}$ & Face 1 & Face 2 & Face 3 & Face 4 & Face 5 & Face 6 & Face 7 & Face 8 & Face 9 \\
\hline 1 & 6575.89 & 6865.27 & 3196.66 & 1816.162 & 1398.12 & 1137.18 & 2752.34 & 798.47 & 1635.24 \\
\hline 2 & 1115.87 & 867.74 & 897.13 & 830.73 & 816.40 & 1060.80 & 6547.87 & 816.11 & 1758.06 \\
\hline 3 & 1509.26 & 1322.38 & 1132.46 & 846.23 & 1227.64 & 6680.36 & 1605.06 & 1343.65 & 4933.15 \\
\hline 4 & 1033.20 & 1295.46 & 1133.06 & 925.87 & 951.78 & 6942.66 & 8377.96 & 8553.80 & 5729.37 \\
\hline 5 & 831.02 & 1266.25 & 1080.76 & 826.25 & 6773.00 & 3411.72 & 2296.55 & 1153.05 & 9212.70 \\
\hline 6 & 915.20 & 1012.78 & 1126.04 & 824.05 & 820.64 & 852.79 & 7855.39 & 2654.95 & 4705.32 \\
\hline 7 & 904.68 & 816.20 & 870.61 & 823.37 & 806.54 & 4184.95 & 870.52 & 813.93 & 814.66 \\
\hline 8 & 971.35 & 855.15 & 976.11 & 817.09 & 950.79 & 818.30 & 902.94 & 818.20 & 819.93 \\
\hline 9 & 901.76 & 831.25 & 889.93 & 900.24 & 900.59 & 819.94 & 823.06 & 1011.00 & 960.84 \\
\hline 10 & 830.97 & 823.87 & 901.44 & 848.54 & 1851.98 & 864.03 & 835.12 & 973.43 & 948.34 \\
\hline 11 & 832.28 & 869.25 & 913.56 & 866.51 & 889.02 & 1005.80 & 966.75 & 1499.73 & 847.55 \\
\hline 12 & 819.63 & 860.48 & 965.04 & 913.38 & 818.14 & 919.31 & 937.65 & 870.19 & 884.35 \\
\hline 13 & 1001.42 & 846.14 & 818.84 & 2821.5 & 817.88 & 1025.73 & 855.15 & 858.94 & 821.62 \\
\hline 14 & 1424.40 & 864.74 & 853.94 & 816.37 & 850.78 & 832.70 & 849.02 & 816.61 & 899.64 \\
\hline 15 & 818.18 & 823.99 & 821.93 & 944.27 & 1001.46 & 823.36 & 900.54 & 816.35 & 848.95 \\
\hline 16 & 921.95 & 837.84 & 1058.30 & 972.45 & 819.52 & 901.47 & 825.33 & 968.29 & 915.40 \\
\hline 17 & 899.33 & 1045.80 & 824.01 & 857.66 & 965.53 & 823.64 & 828.59 & 832.33 & 1063.82 \\
\hline 18 & 1536.84 & 984.36 & 845.18 & 969.27 & 977.60 & 1440.4 & 1068.5 & 936.11 & 964.81 \\
\hline 19 & 1234.40 & 979.99 & 964.17 & 825.51 & 1037.4 & 818.61 & 874.14 & 1047.76 & 966.43 \\
\hline 20 & 890.07 & 929.83 & 919.91 & 968.92 & 817.83 & 964.79 & 917.45 & 819.08 & 982.88 \\
\hline
\end{tabular}


International Journal on Soft Computing (IJSC) Vol.7, No. 4, November 2016

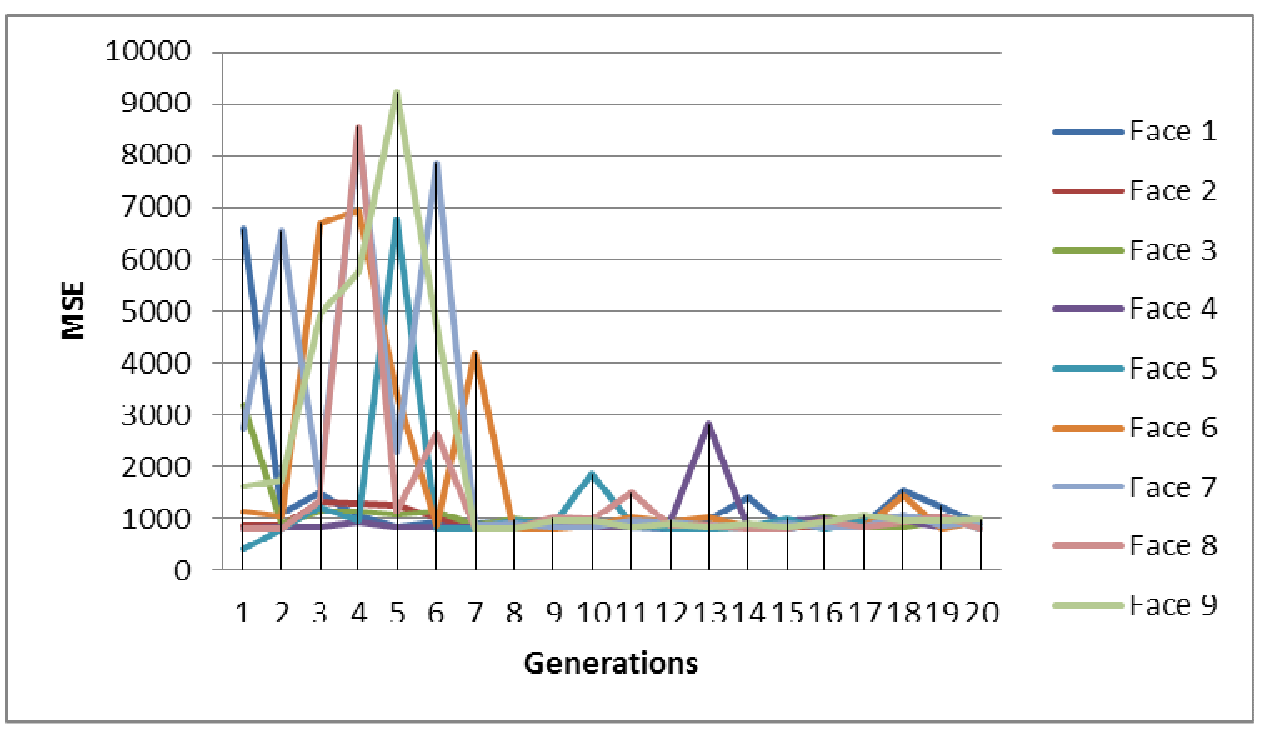

Figure 7: Graph of MSE (Young Population)

Table 3: Final Population after 20 Generation (Old Population)

\begin{tabular}{|l|l|l|l|l|l|l|l|l|l|}
\hline $\begin{array}{l}\text { Face } \\
\text { Gen }\end{array}$ & Face 1 & Face 2 & Face 3 & Face 4 & Face 5 & Face 6 & Face 7 & Face 8 & Face 9 \\
\hline 1 & 1431.23 & 1153.11 & 2878.64 & 511.15 & 1146.86 & 4914.74 & 1308.75 & 1461.32 & 2698.54 \\
\hline 2 & 1257.42 & 1112.41 & 908.86 & 1130.82 & 2014.21 & 1153.73 & 1256.49 & 8873.04 & 2772.91 \\
\hline 3 & 1120.96 & 1816.36 & 1076.51 & 1139.53 & 1145.32 & 1134.93 & 1194.01 & 4743.95 & 9060.10 \\
\hline 4 & 4462.25 & 1133.22 & 940.66 & 1131.19 & 1143.41 & 1143.38 & 1143.11 & 2201.80 & 1538.10 \\
\hline 5 & 1144.10 & 1087.46 & 1019.95 & 1127.60 & 1134.24 & 1126.31 & 1160.80 & 1147.81 & 1146.19 \\
\hline 6 & 4000.30 & 1202.86 & 1138.55 & 1130.76 & 1015.02 & 143.60 & 2133.07 & 1143.55 & 1108.77 \\
\hline 7 & 1125.39 & 1675.82 & 1142.28 & 1078.75 & 1123.78 & 1109.05 & 1070.18 & 1108.72 & 1836.99 \\
\hline 8 & 1125.97 & 1142.91 & 1109.42 & 1123.56 & 1108.95 & 1140.99 & 1127.38 & 1122.18 & 1227.56 \\
\hline 9 & 1143.67 & 1110.93 & 1129.70 & 1391.66 & 2553.60 & 1118.98 & 1036.19 & 1113.98 & 1141.56 \\
\hline 10 & 1315.29 & 1043.99 & 1115.83 & 1083.91 & 1107.89 & 1124.31 & 1101.20 & 1013.83 & 1062.34 \\
\hline 11 & 1145.15 & 1138.30 & 1018.18 & 1016.39 & 3091.19 & 1145.21 & 1124.62 & 1134.60 & 1109.93 \\
\hline 12 & 1015.18 & 1114.12 & 1025.80 & 1064.26 & 1132.74 & 1112.51 & 1108.57 & 1113.12 & 1013.46 \\
\hline 13 & 1112.33 & 2323.08 & 1108.53 & 1111.34 & 1399.08 & 1049.77 & 2020.83 & 1141.52 & 3234.54 \\
\hline 14 & 1133.04 & 1015.27 & 1144.83 & 1117.88 & 2983.03 & 1108.11 & 1141.82 & 1145.14 & 1106.03 \\
\hline 15 & 1926.40 & 1577.58 & 1140.37 & 1048.10 & 1135.61 & 1017.96 & 1297.80 & 1070.23 & 1129.32 \\
\hline 16 & 1108.45 & 1144.62 & 1107.96 & 1141.11 & 1167.96 & 2102.49 & 1108.62 & 1117.61 & 1108.17 \\
\hline 17 & 1022.07 & 1022.39 & 2972.77 & 1143.40 & 1099.93 & 1009.77 & 1141.56 & 1108.73 & 1107.82 \\
\hline 18 & 1113.68 & 1095.35 & 1132.13 & 1106.48 & 1131.28 & 1144.20 & 3562.65 & 1490.80 & 1144.87 \\
\hline 19 & 1108.46 & 1011.43 & 1122.13 & 1061.04 & 1118.53 & 1127.44 & 1125.32 & 1111.99 & 1121.25 \\
\hline 20 & 1130.77 & 1086.23 & 1108.90 & 1137.04 & 1082.53 & 1119.67 & 1133.39 & 1129.49 & 1121.43 \\
\hline
\end{tabular}




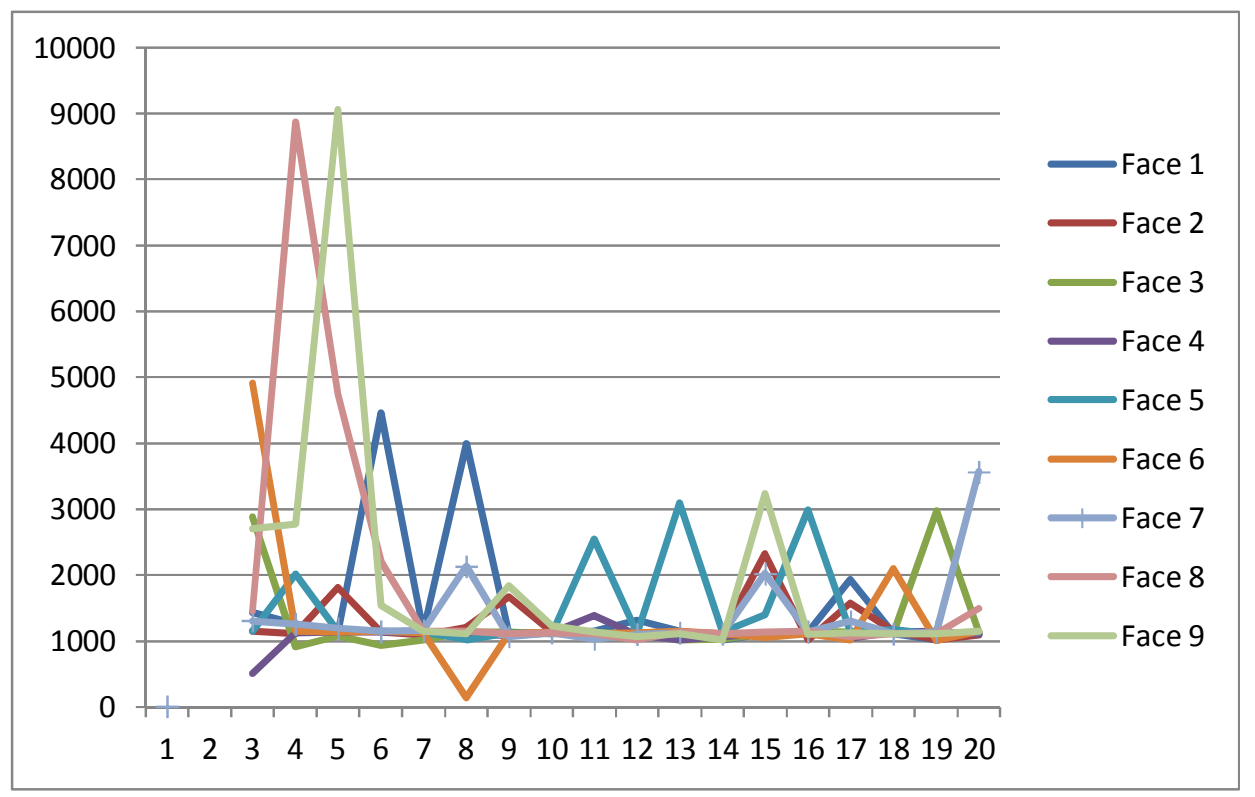

Figure 8: Graph of MSE (Old Population)

\section{CONCLUSIONS}

In this paper, we have performed the experiment for face sketch generation based on evolutionary genetic algorithm. The face is described using facial feature description such as gender, age and shape. The face other features such as size of left eye, right eye, left eyebrow, right eye brow, nose and mouth are captured through the graphical user interface as shown in Figure 3. The face sketch generation using face description based on genetic algorithm is novelty concept implemented in paper. Genetic algorithm works on the population of face and evolves through several generations. In each generation, the faces with higher fitness value retained and the faces with the lower fitness value are removed. Thus, genetic algorithm iterates through the several generation until the desired face is generated.

\section{REFERENCE}

[1] Charlie D Frowd, "Implement Holistic Dimensions for a Facial Composite system" Journal of Multimedia Vol. 1 no 3 June 2006.

[2] Douglas DeCarlo, Dimitris Metaxas, Mathew Stone, "An Anthropometric Face Model using Variational Techniques", Proceedings SIGGRAPH 98.

[3] Fan Yang "Non-parametric Generation Techniques of Example-based Human Facial sketch", Proceedings of the fifth world Congress on Intelligent control and Automation, June 15-19 2004 Hangzhou, P R China.

[4] Futoshi Sugimoto "A Human Interface to search and draw facial images in Mind by Using Psychometrical model of faces", IEEE International Fuzzy Systems Conference Proceedings august 22-25 1999 Seoul Korea.

[5] Hong Chen Ying-Qing Xu, Heung, "Example -based Facial Sketch Generation with nonparametric sampling", IEEE 2001.

[6] Hao Wang, Kangqiao Wang, "Facial Feature Extraction and Image-Based Face Drawing", International conference on Signal Processing", Proceedings 2002

[7] Hao Wang, "Image-Based Face Drawing Using Active Shape and Parametric Morphing" ,IEEE International conference on Neural Network and Signal Processing Nanjing, China, December 1417, 2003. 
[8] Hong Chen Ziqiang Liu, Chunk Rose, "Example -Based Composite Sketching of human portraits", Proceedings of Third International Symposium NPAR 2004

[9] Hong Chen, Lin Liang Yinng,"Example -based Automatic Portraiture", The fifth Asian Conference on Computer Vision 23-25 January 2002, Melbourne , Australia.

[10] Junji Nishino, Tomonori Kameyama, "Linguistic Knowledge Acquisition system on Facial Caricature Drawing System"

[11] Mayada F Abdual Halim, Hussein Hamdi AI-Fiadh, "Facial Composite System Using Genetic Algorithm", Proceedings of the international on Computer Graphics , Imaging and Visualization (CGIV'06)

[12] Masashi Yamada, Takehisa Onisawa, "Logo drawing System Applying Interactive Genetic Algorithms", IEEE 2006

[13] Pong C, Yuen , C H Man, "Human Face Image Searching System Using Sketches", IEEE Transaction on Systems man and cybernetics -part A : system Humans , Vol. 32 ,No. 4 July 2007.

[14] Pualak Purkit, Bhabatosh Chanda, Shrikant Kulkarni, "A Novel Technique for Sketch to Photo Synthesis"

[15] Shino Iwashita, Takehisa Onisawa, "A Study on Facial Caricature Drawing by fuzzy Theory", IEEE 1997.

[16] Stuart Gibson "Synthesis of Photographic Quality Facial composite using Evolutionary Algorithms" In proceeding's of British Machine Vision Conference 2003.

[17] Sylvain Calinon , Julien Epiney , Aude Billard, "A Humanoid Robot Drawing Human Portraits", Proceedings of 2005 fifth IEEE-RAS International Conference of Humanoid Robots.

[18] Takesisa Onisawa, Yuske Hirasawa, "Facial caricature Drawing Using subjective Image of a Face Obtained By words", IEEE 2004.

[19] Xiaoou Tang, Xiaogang Wang,"Face Sketch Synthesis and Recognition”, Proceedings of the Ninth IEEE International Conference on Computer Vision (ICCV 2003).

[20] Xiaoou Tang, Xiaogang Wang, "Face Sketch Recognition", IEEE Transactions on Circuits and System for Video Technology, Vol. 14 No 1, Jan 2004.

[21] Melanie Mitchell "An Introduction to Genetic Algorithms", Printice-Hall of India Private Limited , New Delhi, pp. 35-45, 1998.

[22] http://mmlab.ie.cuhk.edu.hk/archive/facesketch.html

[23] http://www.vision.caltech.edu/html-files/archive.htm

\section{Authors}

N K Bansode currently doing research in computer science in university of Pune. He has published a number of papers in the different international conferences and journals

P K Sinha is a researcher, scientist, inventor, IEEE Fellow and the internationally acclaimed author of computer textbooks, with more than twenty-five years of professional experience. 